\title{
EDITORIAL
}

\section{Potentials for Use of Medicinal Plants in Female Reproductive Disorders - The Way Forward}

\author{
DOI: $10.29063 /$ ajrh2017/v21i4.1
}

\section{Enitome E Bafor}

Department of Pharmacology \& Toxicology, University of Benin, Benin-City, Edo State, Nigeria

*For Correspondence: Email: enitome.bafor@uniben.edu

\section{Introduction}

In the past years, the use of traditional medicine has gained much recognition worldwide, although more engrained in some cultures than others. This form of medicine relies on the use of certain herbal plants (medicinal plants) and other remedies for beneficial biological effects ${ }^{1}$. In Africa and Asia there appears to be a high reliance on medicinal plants particularly by people of lower income and this has been attributed partly to the often unreachable cost of allopathic drugs, unavailability of modern health care facilities, and also the cultural acceptability of the traditional system ${ }^{2}$. At the same time, there are some fields of thought that appear to disregard or discountenance the importance and use of medicinal plants. Such thoughts may have arisen for several reasons including, the idea that medicinal plant use is mainly for low income earners, that the use of medicinal plants have little to offer, and that medicinal plants have no activity and act more like a placebo. Evidence from scientific research worldwide has however proven that medicinal plants have immense biological and health applicability. Some of the earliest drugs were first discovered from traditionally used plants prior to their availability as synthesized drugs ${ }^{3}$. Medicinal plants should therefore be accorded due consideration and support across different health and scientific disciplines.

The use of medicinal plants has found application in several diseases and health conditions and reproductive disorders are not left out. In the traditional system of medicine, plant preparations in the forms of macerations, tinctures, concoctions, or infusions are used for a wide range of diseases. The application of medicinal plants to female reproductive health issues is gaining interest, as reproductive disorders are considered an important public health and social problem ${ }^{4}$. In developing countries, particularly in sub-Saharan Africa, reproductive health disorders pose a major burden ${ }^{5,6}$ and are considered the second most prevalent health care problem in Africa ${ }^{7}$.

The starting point for use of plants often begins from traditional healers and natives of a particular culture. The plants utilized vary from culture to culture which is not surprising owing to the vast varieties of plants in existence and also to the varying localization of plants from one geographical region to another. Plants collected may include species with known biological activity on reproductive disorders for which active compound(s) have not been isolated. On collection of plants, phytochemists (natural product chemists) prepare the extracts from the plant materials, which are subjected to biological screening using pharmacologically relevant assays, and then proceed to the isolation and characterization of the active compound(s) through bioassay-guided fractionation. Medicinal plants have played an important role of providing new chemical entities through the years even so for female reproductive health issues ${ }^{8}$. Detailed ethnobotanical surveys have been reported for several native communities around the world and many traditionally-used plants or remedies have been identified. The knowledge and the correct use of these natural medicines has been acquired and improved over many generations. Documentation of traditional knowledge of medicinal plants is crucial, since it provides chemists and pharmacologists with starting points for "targeted" analysis, discovery of novel remedies, and natural drugs for the treatment of pregnancy and birth-related problems.

\section{Some medicinal plants used in female reproductive health}

Medicinal plants can be used for their beneficial effects on many female reproductive processes ranging from pregnancy, to labour induction, elimination of retained placenta, and management of post-partum haemorrhage. Most often the biological effects elicited 
by these remedies are due to secondary metabolites (small chemicals, peptides or proteins) that primarily act on the reproductive system. The nature of these actions may involve the modulation of uterine contractions at labour, resulting in either the stimulation or inhibition of myometrial muscle contractions, or may modulate other reproductive processes such as folliculogenesis, and reproductive hormone(s) regulation.

Medicinal plants have found application in several female reproductive disorders. Ethnobotanical studies are additionally required to identify and document the native use of plants to treat gynaecological health issues ${ }^{1}$. Some studies provide initial pharmacological characterisation ${ }^{9,10}$. Ficus exasperata (Moraceae) for instance in Nigeria has been reported to elaborate constituents which are able to manage preterm labour and dysfunctional labour ${ }^{11}$. Preterm labour is currently the single largest cause of maternal and perinatal mortality in developed countries and a major contributor to childhood developmental problems $^{12,13}$. Currently used intervention therapies to suppress uterine contractions and delay labour, have harmful side effects for mother and baby ${ }^{14}$. Thus, there is a need for treatments that are effective in reducing the premature birth and/or providing for longer gestation, with better safety and tolerability profiles. Uterotonic or oxytocic drugs are used to manage dysfunctional labour and new drugs for dysfunctional labour still remain largely unsearched. There is therefore evidently an increasing need to search for new chemical entities from medicinal plants which till date still remain largely untapped. Emilia coccinea (Asteraceae) and Alchornea laxiflora (Euphorbiaceae) also in Nigeria have shown usefulness as potent contraceptive agents ${ }^{15,16}$. In native Northern America, raspberry leaves are taken as tonics during pregnancy to prepare for labour, black haw is taken to prevent miscarriage and blue cohosh is taken to induce labour ${ }^{17}$.

Preparations of black cohosh root (Actaea racemosa [Nutt.] L.), Goldenseal root (Hydrastis canadensis L.), and Chaste tree fruits (Vitex agnuscastus L.) are listed in the U. S. Pharmacopoeia and are available as dietary supplements to be used for premenstrual stress syndrome, as emmenagogue agents, and for gynaecological problems. Castor oil (Ricinus communis L.) $)^{18}$ and cotton bark root (Gossypium hirsutum L. $)^{19}$ are utilized by midwives for applications during pregnancy and labour ${ }^{20,21}$. In Germany aerial parts of marjoram (Origanum majorana L.), lime (Tilia cordata Mill. and Tilia platyphyllos Scop.) as well as chamomile (Matricaria recutita L.), and fruits of caraway (Carum carvi L.) are used to induce labour ${ }^{22}$.
There is a vast array of information available on traditionally used herbs to treat gynaecological problems. The medicinal properties and mode of action of many of these plants have not yet been studied in molecular detail but they may affect a number of different physiological targets and pathways in the female body.

\section{Safety concerns with medicinal plants use}

Regardless of the acceptability by many of the purported safety in use of herbal medicines, some of these medicines may have harmful side effects and when taken in large unregulated quantities may lead to the death of the unborn baby and/or uterine rupture, and other longer-term effects on the mother or baby. This necessitates detailed studies to identify the correct dosage that is safe to use, and hence significant caution must be applied on the arbitrary use of medicinal plant.

\section{Importance of identification, documentation and preservation of medicinal plants}

Another major concern however, is that traditional medicinal knowledge and its associated plants, may be lost if care is not taken to adequately document and preserve this knowledge and the plants of importance. This is particularly important since knowledge of most of these medicinal plants are simply handed down by word of mouth over successive generations ${ }^{23}$. There is also the issue of environmental degradation, deforestation, over-exploitation, over-grazing, agricultural land expansion, and acculturation which continue to threaten the existence of these medicinal plants ${ }^{23}$. The need therefore to investigate and document traditional knowledge of medicinal plants which will promote proper utilization of these medicinal plants is highly encouraged. This will also enhance the promotion and protection of indigenous medicinal plant knowledge of any community as a vital part of the nation's heritage.

The issue of secrecy and intellectual property rights amongst traditional medical care givers has made it difficult for them to disclose freely the actual medicinal plant, proportions and combinations used for treatment of specific diseases. This needs to be resolved for meaningful identification and documentation of medicinal plants used within communities.

\section{Conclusion and Way Forward}

In conclusion, medicinal plants are an essential component of drug discovery for female reproductive disorders. There is a great need for new, potent, 
selective and nontoxic therapeutic agents (agonists and antagonists) that modulate the female reproductive system. Traditionally used herbal medicines and their active ingredients, are ideal starting points for biological target-oriented drug discovery efforts for gynaecological disorders. It is vital to incorporate this form of medicine to improve gender equity in basic health care provision and national development through indigenous knowledge innovations and bioprospecting.

A trusted regulatory body should be established to oversee all intellectual property rights of individuals with documented traditional herbal plants and their usage. Their rights and ownership of such formulation should be respected to curtail secrecy, and royalties should be paid to them when drugs are later developed from these plants.

Furthermore, documented plant species will assist in the conservation of such relevant plant species for female reproductive health issues and may lead to the isolation of useful ingredients to produce drugs and other medicinal consumables. Health care givers, scientists and policy makers are therefore enjoined to support research and documentation of medicinal plants use in reproductive disorders.

\section{References}

1. Gruber CW and O'Brien M. Uterotonic plants and their bioactive constituents. Planta Med 2011; 77: 207-220.

2. Ross S and Maxwell S. Prescribing and the core curriculum for tomorrow's doctors: BPS curriculum in clinical pharmacology and prescribing for medical students. $\mathrm{Br} J$ Clin Pharmacol 2012; 74: 644-661.

3. Balick MJ and Cox PA. Plants, People, and Culture: the Science of Ethnobotany. New York, NY: Scientific American Library, 1997.

4. Diame GLO. Ethnobotany and ecological studies of plants used for reproductive health: A case study at Bia Biosphere reserve in the western region of Ghana. Accra, Ghana, 2010.

5. Nordeng H, Al-Zayadi W, Diallo D, Ballo N and Paulsen BS. Traditional medicine practitioners' knowledge and views on treatment of pregnant women in three regions of Mali. J Ethnobiol Ethnomed 2013; 9: 67.

6. Kamatenesi MM, Acipa A and Oryem-Origa H. Medicinal plants of Otwal and Ngai Sub Counties in Oyam District, Northern Uganda. J Ethnobiol Ethnomed 2011; 7: 7.

7. World Health Organization. Progress Report Reproductive health strategy to accelerate progress towards the attainment of international development goals and targets. Geneva. Epub ahead of print 2010. DOI: 10.1016/S09688080(05)25166-2.Accessed 20 $0^{\text {th }}$, November 2017.

8. Bafor EE, Rowan EG and Edrada-Ebel R. Metabolomics-
Coupled Functional Pharmacology of Chlorophyll Compounds Isolated From the Leaves of Ficus Exasperata Vahl (Moraceae) Provides Novel Pathways on Myometrial Activity. Reprod Sci 2017; (Online first).

9. Desta B. Ethiopian traditional herbal drugs. Part III: Antifertility activity of 70 medicinal plants. $J$ Ethnopharmacol 1994; 44: 199-209.

10. Michel J, Duarte RE, Bolton JL, Huang Y, Cáceres A, Veliz M, Soejarto DD and Mahady GB. Medical potential of plants used by the Q'eqchi Maya of Livingston, Guatemala for the treatment of women's health complaints. J Ethnopharmacol 2007; 114: 92-101.

11. Bafor EE, Lim C V., Rowan EG, and Edrada-Ebel R. The leaves of Ficus exasperata Vahl (Moraceae) generates uterine active chemical constituents. J Ethnopharmacol 2013; 145: 803-812.

12. Slattery MM and Morrison JJ. Preterm delivery. Lancet 2002; 360: 1489-1497.

13. World Health Organization. World health report 2005: make every mother and child count. Epub ahead of print 2005. DOI: ISBN 9241562900 (NLM).

14. Higby K, Xenakis EMJ and Pauerstein CJ. Do tocolytic agents stop preterm labor? A critical and comprehensive review of efficacy and safety. American Journal of Obstetrics and Gynecology 1993; 168: 1247-1259.

15. Elvis-Offiah UB, Bafor EE, Eze GI, Igbinumwen O,

Viegelmann $C$ and Edrada-Ebel R. In vivo investigation of female reproductive functions and parameters in nonpregnant mice models and mass spectrometric analysis of the methanol leaf extract of Emilia Coccinea (Sims) G Dons. Physiol Rep 2016; 4: e13047.

16. Bafor EE, Eyohan SE, Omoruyi O, Elvis-Offiah UB, Ayinde $\mathrm{B}$, Eze GI, Okosun IV, Obarisiagbon PA, Igbinuwen O and Braimoh KP.. Preliminary endocrinological, histological and haematological investigation of Alchornea laxiflora (Euphorbiaceae) leaf extract effects on the ovary, uterus and cervix of mouse models. $J$ Sci Pract Pharm 2016; 2: 55-63.

17. Westfall RE. Herbal medicine in pregnancy and childbirth. Adv Ther 2001; 18: 47-55.

18. Kelly AJ and Kavanagh J, Thomas J. Castor oil, bath and/or enema for cervical priming and induction of labour. Cochrane database Syst Rev 2013; 7: CD003099.

19. Nissim R. Natural healing in gynaecology: a manual for women. In: Pandora's Health. London: Thorsons Publishers, 1996, p. 220.

20. Bayles BP. Herbal and Other Complementary Medicine Use by Texas Midwives. J Midwifery Women's Heal 2007; 52: 473-478.

21. Born D and Barron ML. Herb use in pregnancy: what nurses should know. MCN Am J Matern Child Nurs 2005; 30: 201-208.

22. Gruber CW and O'Brien M. Uterotonic plants and their bioactive constituents. Planta Med 2011; 77: 207-20.

23. Tsobou R, Mapongmetsem PM and Van Damme P. Medicinal Plants Used for Treating Reproductive Health Care Problems in Cameroon, Central Africa1. Econ Bot 2016; 70: $145-159$. 


\section{EDITORIAL}

\section{Potentiels d'utilisation des plantes médicinales dans les troubles de la reproduction chez la femme - La voie à suivre}

DOI: $10.29063 / a j r h 2017 / v 21 \mathrm{i} 4.1$

Enitome E Bafor

Département de Pharmacologie et de Toxicologie, Université de Bénin, Benin-City, État d'Edo, Nigéria

* Pour Correspondance : Email: enitome.bafor@uniben.edu

\section{Introduction}

Au cours de ces dernières années, l'utilisation de la médecine traditionnelle a gagné beaucoup de reconnaissance dans le monde entier, bien qu'elle soit plus ancrée dans certaines cultures que dans d'autres. Cette forme de médecine repose sur l'utilisation de certaines plantes médicinales (plantes médicinales) et d'autres remèdes pour des effets biologiques bénéfiques ${ }^{1}$. En Afrique et en Asie, il semble y avoir une forte dépendance des plantes médicinales, en particulier chez les personnes à faible revenu, en partie attribuable au coût souvent inaccessible des médicaments allopathiques, à la indisponibilité des établissements de santé modernes et à l'acceptabilité culturelle des médicaments du système traditionnels ${ }^{2}$. En même temps, certains domaines de pensée semblent ignorer ou décourager l'importance et l'utilisation des plantes médicinales. De telles pensées peuvent avoir surgi pour plusieurs raisons comprenant, l'idée que l'utilisation de plantes médicinales est principalement pour ceux qui gagnent les bas salaires, que l'utilisation de plantes médicinales a peu à offrir, et aussi que les plantes médicinales n'ont aucune activité et agissent plus comme un placebo. Les preuves provenant de la recherche scientifique dans le monde entier ont cependant prouvé que les plantes médicinales ont une immense applicabilité biologique et sanitaire. Certains des premiers médicaments ont d'abord été découverts à partir de plantes utilisées traditionnellement avant leur disponibilité en tant que médicaments synthétisés ${ }^{3}$. Les plantes médicinales devraient donc être dûment prises en compte et soutenues dans les différentes disciplines scientifiques et sanitaires.

L'utilisation de plantes médicinales a trouvé application dans plusieurs maladies et états de santé, et les troubles de la reproduction ne sont pas exclus. Dans le système traditionnel de la médecine, les préparations de plantes sous forme de macérations, de teintures, de concoctions ou d'infusions sont utilisées pour un large éventail de maladies. L'application de plantes médicinales aux problèmes de santé de la reproduction chez les femmes suscite de l'intérêt, car les troubles de la reproduction sont considérés comme un important problème de santé publique et de société ${ }^{4}$. Dans les pays en développement, en particulier en Afrique subsaharienne, les troubles de santé génésique représentent un fardeau important ${ }^{5,} 6$ et sont considérés comme le deuxième problème de santé le plus répandu en Afrique ${ }^{7}$. Le point de départ pour l'utilisation des plantes commence souvent par les guérisseurs traditionnels et les natifs d'une culture particulière. Les plantes utilisées varient d'une culture à l'autre, ce qui n'est pas surprenant en raison des vastes variétés de plantes existantes et de la localisation variable des plantes d'une région géographique à l'autre. Les plantes collectées peuvent inclure des espèces ayant une activité biologique connue sur des troubles de la reproduction pour lesquels les composés actifs n'ont pas été isolés. Lors de la collecte des plantes, les phytochimistes (chimistes de produits naturels) préparent les extraits des plantes, qui sont soumis à 
un criblage biologique en utilisant des dosages pharmacologiquement pertinents, puis procèdent à l'isolement et à la caractérisation des composés actifs par fractionnement par bioessai. Les plantes médicinales ont joué un rôle important en fournissant de nouvelles entités chimiques au cours des années, même pour les problèmes de santé de la reproduction des femmes ${ }^{8}$. Des études ethnobotaniques détaillées ont été rapportées pour plusieurs communautés autochtones dans le monde et de nombreuses plantes ou remèdes traditionnellement utilisés ont été identifiés. La connaissance et l'utilisation correcte de ces médicaments naturels ont été acquises et améliorées sur plusieurs générations. La documentation des connaissances traditionnelles sur les plantes médicinales est cruciale car elle fournit aux chimistes et aux pharmacologues des points de départ pour l'analyse " ciblée », la découverte de nouveaux remèdes et de médicaments naturels pour le traitement de la grossesse et des problèmes liés à l'accouchement.

\section{Certaines plantes médicinales utilisées dans la santé de la reproduction des femmes}

Les plantes médicinales peuvent être utilisées pour leurs effets bénéfiques sur de nombreux processus de reproduction chez la femme, depuis la grossesse jusqu'à l'induction $\mathrm{du}$ travail, l'élimination de la rétention placentaire et la gestion de l'hémorragie post-partum. Le plus souvent, les effets biologiques induits par ces remèdes sont dus à des métabolites secondaires (petits produits chimiques, peptides ou protéines) qui agissent principalement sur le système de la reproduction. La nature de ces actions peut impliquer la modulation des contractions utérines au travail, entraînant soit la stimulation ou l'inhibition des contractions musculaires du myomètre, ou peut moduler d'autres processus de reproduction tels que la folliculogenèse et la régulation des hormones de reproduction.

Les plantes médicinales ont trouvé une application dans plusieurs troubles de la reproduction chez la femme. Des études ethnobotaniques sont en outre nécessaires pour identifier et documenter l'utilisation native des plantes pour traiter les problèmes de santé gynécologiques ${ }^{1}$. Certaines études fournissent une caractérisation pharmacologique initiale ${ }^{9,10}$. Ficus exasperata (Moraceae), par exemple au Nigeria a été signalé pour élaborer des constituants qui sont capables de gérer le travail prématuré et travail fonctionnel ${ }^{11}$. Le travail prématuré est actuellement la principale cause de mortalité maternelle et périnatale dans les pays développés et un contributeur majeur aux problèmes de développement de l'enfant ${ }^{12,13}$. Les thérapies d'intervention actuellement utilisées pour supprimer les contractions utérines et retarder le travail ont des effets secondaires néfastes pour la mère et le bébé ${ }^{14}$. Ainsi, il y a la nécessité pour des traitements qui soient efficaces pour réduire l'accouchement prématuré et / ou assurer une gestation plus longue, avec de meilleurs profils d'innocuité et de tolérace. Des médicaments utérotoniques ou ocytociques sont utilisés pour gérer le travail dysfonctionnel et de nouveaux médicaments pour le travail dysfonctionnel restent encore largement non recherchés. Il est donc manifestement de plus en plus nécessaire de rechercher de nouvelles entités chimiques à partir de plantes médicinales qui, jusqu'à ce jour, restent largement inexploitées.

Emilia coccinea (Asteraceae) et Alchornea laxiflora (Euphorbiaceae) également au Nigéria ont montré leur utilité en tant qu'agents contraceptifs puissants ${ }^{15,16}$. Dans l'Amérique du Nord indigène, les feuilles de framboisier sont prises comme toniques pendant la grossesse pour se préparer au travail, la viande noire est prise pour prévenir les fausses couches et l'actée à grappes bleues est utilisée pour induire le travail ${ }^{17}$. Les préparations de racine d'actée à grappes noires (Actaea racemosa [Nutt.] L.), de racine d'hydraste du Canada (Hydrastis canadensis L.) et de gattilier (Vitex agnus-castus L.) figurent dans la Pharmacopée américaine et sont disponibles sous forme de compléments alimentaires et utilisé pour le syndrome de stress prémenstruel, $m$ quand les médicaments sont développés plus tard à partir de 
ces plantes. En outre, des espèces végétales documentées aideront à la conservation de ces espèces végétales pertinentes pour les problèmes de santé reproductive des femelles et peuvent conduire à l'isolement d'ingrédients utiles pour produire des médicaments et autres consommables médicinaux. Les fournisseurs de soins de santé, les scientifiques et les décideurs sont donc encouragés à soutenir la recherche et la documentation sur l'utilisation des plantes médicinales dans les troubles de la reproduction comme agents emmenagogue, et pour les problèmes gynécologiques. L'huile de ricin (Ricinus communis L. $)^{18}$ et la racine d'écorce de coton (Gossypium hirsutum L.) ${ }^{19}$ sont utilisées par les sages-femmes pour des applications pendant la grossesse et le travail ${ }^{20,21}$. En Allemagne, des parties aériennes de la marjolaine (Origanum majorana L.), de la chaux (Tilia cordata Mill et Tilia platyphyllos Scop.) Ainsi que de la camomille (Matricaria recutita $\mathrm{L}$.) et des fruits du carvi (Carum carvi L.) sont utilisées pour induire le travail ${ }^{22}$. Il existe une vaste gamme d'informations sur les herbes utilisées traditionnellement pour traiter les problèmes gynécologiques. Les propriétés médicinales et le mode d'action de plusieurs de ces plantes n'ont pas encore été étudiés dans les détails moléculaires, mais ils peuvent affecter un certain nombre de cibles physiologiques et de voies différentes dans le corps féminin.

\section{Les préoccupations de sécurité avec les plantes médicinales}

Certains de ces médicaments peuvent avoir des effets secondaires néfastes et, lorsqu'ils sont pris en grandes quantités non réglementées, ils peuvent entraîner la mort du bébé à naître et / ou la rupture de l'utérus et d'autres effets à long terme sur la mère ou le bébé. Cela nécessite des études détaillées pour identifier le dosage correct qui est sûr à utiliser, et donc une grande prudence doit être appliquée sur l'utilisation arbitraire de la plante médicinale.
Importance de l'identification, de la documentation et de la préservation des plantes médicinales

Une autre préoccupation majeure est cependant que les connaissances médicinales traditionnelles et leurs plantes associées peuvent être perdues si l'on ne prend pas soin de documenter et préserver ces connaissances et les plantes importantes. Ceci est particulièrement important puisque la connaissance de la plupart de ces plantes médicinales est simplement transmise oralement au cours des générations successives ${ }^{23}$. Il y a aussi la question de la dégradation de l'environnement, de la déforestation, de la surexploitation, du surpâturage, de l'expansion des terres agricoles et de l'acculturation qui continuent de menacer l'existence de ces plantes médicinales ${ }^{23}$. La nécessité d'étudier et de documenter les connaissances traditionnelles sur les plantes médicinales qui favoriseront une utilisation adéquate de ces plantes médicinales est donc fortement encouragée. Cela renforcera également la promotion et la protection de la connaissance des plantes médicinales indigènes de toute communauté en tant que partie essentielle du patrimoine de la nation. La question du secret et des droits de propriété intellectuelle entre les fournisseurs des soins médicaux traditionnels a rendu difficile pour eux de divulguer librement la plante médicinale réelle, les proportions et les combinaisons utilisées pour le traitement des maladies spécifiques. Cela doit être résolu pour une identification et une documentation significative des plantes médicinales utilisées dans les communautés.

\section{Conclusion et Perspectives}

En conclusion, les plantes médicinales sont une composante essentielle de la découverte des médicaments pour les troubles de la reproduction chez la femme. Il y a un grand besoin de nouveaux agents thérapeutiques puissants, sélectifs et non 
toxiques (agonistes et antagonistes) qui modulent le système de la reproduction chez la femme. Les médicaments à base de plantes traditionnellement utilisés et leurs ingrédients actifs sont des points de départ idéaux pour les efforts de découverte des médicaments orientés vers des cibles biologiques pour les troubles gynécologiques. Il est essentiel d'incorporer cette forme de médecine pour améliorer l'équité entre les sexes dans la fourniture de soins de santé de base et le développement national grâce à des innovations de connaissances indigènes et à la bioprospection.

Un organisme de confiance doit être créé pour surveiller tous les droits de propriété intellectuelle des plantes et leur usage. Leurs droits et la propriété d'une telle formulation devraient être respectés afin de restreindre le secret, et des redevances devraient être versées quand les médicaments sont élaborés plus tard à partir de ces plantes.

En outre, des espèces végétales documentées aideront à la conservation de ces espèces végétales pertinentes pour les problèmes de santé de la reproduction des femmes et peuvent conduire à l'isolement d'ingrédients utiles pour produire des médicaments et autres consommables médicinaux. Les fournisseurs de soins de santé, les scientifiques et les décideurs sont donc encouragés à soutenir la recherche et la documentation sur l'utilisation des plantes médicinales dans les troubles de la reproduction.

\section{Références}

1. Gruber $\mathrm{CW}$ et $\mathrm{O}^{\prime}$ Brien $\mathrm{M}$. Les plantes utérotoniques et leurs constituants bioactifs. Planta Med 2011; 77: 207-220.

2. Ross S et Maxwell S. Prescription et le programme de base pour les médecins de demain: programme d'études BPS en pharmacologie clinique et prescription pour les étudiants en médecine. $\mathrm{Br} J$ Clin Pharmacol 2012; 74: 644-661.

3. Balick MJ et Cox PA. Plants, People and Culture: the Science of Ethnobotany. New York, NY: Scientific American Library, 1997.

4. Diame GLO. Ethnobotany and Ecological Studies of Plants used for Reproductive Health: A Case Study at Bia Biosphere Reserve in the Western region of Ghana. Accra, Ghana, 2010.
5. Nordeng H, Al-Zayadi W, Diallo D, Ballo N et Paulsen BS. Connaissances et points de vue des praticiens de la médecine traditionnelle sur le traitement des femmes enceintes dans trois régions du Mali. $J$ Ethnobiol Ethnomed 2013; 9: 67.

6. Kamatenesi MM, Acipa A et Oryem-Origa H. Plantes médicinales des sous-comtés d'Otwal et de Ngai dans le district d'Oyam, au nord de l'Ouganda. $J$ Ethnobiol Ethnomed 2011; 7: 7.

7. Organisation mondiale de la santé. Rapport d'avancement Stratégie de santé génésique pour accélérer les progrès vers la réalisation des objectifs et cibles de développement internationaux. Genève. Epub avant impression 2010. DOI: 10.1016 / S0968-8080 (05) 25166-2.Accédé le 20 novembre 2017.

8. Bafor EE, Rowan EG et Edrada-Ebel R. Pharmacologie fonctionnelle couplée à la métabolomique des composés de chlorophylle isolés à partir des feuilles de Ficus Exasperata Vahl (Moraceae) fournit de nouvelles voies sur l'activité myométriale. Reprod Sci 2017; (En ligne d'abord) .

9. Desta B. Drogues à base de plantes traditionnelles éthiopiennes. Partie III: Activité anti-fertilité de 70 plantes médicinales. J Ethnopharmacol 1994; 44: 199-209.

10. Michel J, Duarte RE, Bolton JL, Huang Y, Cáceres A, Véliz M, Soejarto DD et Mahady GB. Potentiel médical des plantes utilisées par les Q'eqchi Maya de Livingston, Guatemala pour le traitement des plaintes de santé des femmes. $J$ Ethnopharmacol 2007; 114: 92-101.

11. Bafor EE, Lim C V., Rowan EG et Edrada-Ebel R. Les feuilles de Ficus exasperata Vahl (Moraceae) génèrent des constituants chimiques actifs de l'utérus. J Ethnopharmacol 2013; 145: 803-812.

12. Slattery MM et Morrison JJ. Livraison prématurée. Lancet 2002; 360: 1489-1497.

13. Organisation mondiale de la santé. Rapport sur la santé dans le monde 2005: faites en sorte que chaque mère et chaque enfant compte. Epub avant impression 2005. DOI: ISBN $924156290 \quad 0$ (NLM).

14. Higby K, Xenakis EMJ et Pauerstein CJ. Les agents tocolytiques arrêtent-ils le travail prématuré? Un examen critique et complet de l'efficacité et de la sécurité. American Journal of Obstetrics and Gynecology 1993; 168: 1247-1259.

15. Elvis-Offiah UB, Bafor EE, Eze GI, Igbinumwen O, Viegelmann $\mathrm{C}$ et Edrada-Ebel R. Étude in vivo des fonctions et des paramètres reproducteurs femelles dans des modèles de souris non-enceintes et analyse spectrométrique de masse de l'extrait de feuilles de méthanol d'Emilia Coccinea (Sims) G Dons. Physiol Rep 2016; 4: e13047.

16. Bafor EE, Eyohan SE, Omoruyi O, Elvis-Offiah UB, 
Ayinde B, Eze GI, Okosun IV, Obarisiagbon PA, Igbinuwen $\mathrm{O}$ et Brahimh KP .. Étude préliminaire endocrinologique, histologique et hématologique de l'extrait de feuilles d'Alchornea laxiflora (Euphorbiaceae) sur le l'ovaire, l'utérus et le col de la souris. J Sci Pract Pharm 2016; 2: 55-63.

17. Westfall RE. La phytothérapie pendant la grossesse et l'accouchement. Adv Ther 2001; 18: 47-55.

18. Kelly AJ et Kavanagh J, Thomas J. Castor huile, bain et I ou lavement pour l'amorçage cervical et l'induction du travail. Base de données Cochrane Syst Rev 2013; 7: CD003099.

19. Nissim R. Natural Healing in Gynaecology : A Manual for Women, Pandora's Health. Londres: Thorsons Publishers, 1996, p. 220.
20. Bayles BP. Utilisation des médicaments à base de plantes et d'autres médicaments complémentaires par Les sages-femmes de Texas. J Midwifery Women's Heal 2007; 52: 473-478.

21. Born D et Barron ML. L'Utilisation d'herbes pendant la grossesse: ce que les infirmières devraient savoir. MCN Am J Matern Child Nurs2005; 30: 201-208.

22. Gruber $\mathrm{CW}$ et $\mathrm{O}^{\prime}$ Brien $\mathrm{M}$. Les plantes utérotoniques et leurs constituants bioactifs. Planta Med 2011; 77: 207-20.

23. Tsobou R, Mapongmetsem PM et Van Damme P.

Plantes médicinales utilisées pour traiter les problèmes de santé génésique au Cameroun, en Afrique centrale. Econ Bot 2016; 70: 145-159. 